\title{
Los anglicismos como préstamos linguísticos que se han incorporado al español de Ecuador
}

\section{Anglicisms as language loans that have been incorporated into ecuadorian spanish}

\author{
Hugo Hernán Romero Rojas ${ }^{1}$ \\ Universidad Nacional de Chimborazo
}

\section{RESUMEN}

Los préstamos lingüísticos, mejor conocidos como anglicismos, han tomado elementos morfológicos del español y se han agregado al idioma español del Ecuador, ocupando la categoría gramatical, para este trabajo, de verbos de la primera conjugación.

PALABRAS CLAVE: elementos morfológicos, verbos, primera conjugación.

\section{SUMMARY}

The linguistic loans, better known as Anglicisms, have taken morphological elements from Spanish and added them to Ecuador's Spanish language, occupying the grammatical category, 1 Hugo Hernán Romero Rojas hromero@unach.edu.ec 0981007230 Universidad Nacional de Chimborazo https://orcid.org/0000-0001-8335-0599 Riobamba- Ecuador for this work, of verbs of the first conjugation.

KEYWORDS: morphological elements, verbs, first conjugation.

\section{DESARROLLO}

A través de este trabajo de revisión bibliográfica se relatará sobre los préstamos lingüísticos, de ahora en adelante anglicismos, conjunto de palabras que se han adherido al idioma español con la categoría gramatical de verbos con sus respectivos elementos morfológicos, tales como: tiempo, modo, persona y número, que participan de la primera conjugación (ar). Como ejemplo tenemos la palabra inglesa blog, su categoría gramatical es sustantivo que adquiere los elementos morfológicos y se transforma en verbo. Un individuo puede decir "blogueo toda mi información", como se puede visualizar blogueo posee el morfema o que concierne a la primera 
persona del singular del modo indicativo, tiempo presente. Este fenómeno lingüístico se presenta en similares condiciones con otras palabras del inglés.

En la actualidad los préstamos lingüísticos de la lengua franca, es decir del inglés, ocupan el primer lugar en los diferentes ámbitos de la sociedad, convirtiéndose los nombres, adjetivos y verbos, en este orden, las categorías gramaticales más anexadas, inmediatamente seguidas de los adverbios, preposiciones y las interjecciones.

Los trabajos investigativos referentes al tema no son abundantes y carecen de una normativa a nivel nacional e internacional que regule la forma cómo los préstamos deben pasar al español. El intercambio comercial y tecnológico con países de habla inglesa ha aumentado rápidamente debido a las conexiones por medio de la internet, que rompe los protocolos de migración a nivel mundial. Gracias a este aporte teórico los profesionales afines a las áreas de Lengua y Literatura contarán con una guía y el vocabulario de los diferentes préstamos que podrán ser socializados con alumnos y público en general.

Como hipótesis se puede plantear que las palabras del inglés que van a desempeñar la categoría gramatical de verbos obtienen los elementos morfológicos de la primera conjugación (ar) y se unen al vocabulario como palabras recién creadas.

Las investigaciones que tratan sobre los préstamos lingüísticos en el Ecuador no han sido desarrollados en un gran número, seguramente porque la investigación cuantitativa predomina sobre la cualitativa, a pesar de ello, existen trabajos que guardan relación, así tenemos la investigación con el título "La actitud lingüística hacia los anglicismos en el registro coloquial quiteño en los estudiantes de primer semestre", se concluyó que el uso de los préstamos fue muy frecuente por parte de los estudiantes. (Cuéllar \& Izurieta, 2017)

Otra investigación titulada "La influencia del inglés en la publicidad escrita en la ciudad de Quito y el impacto que genera en los jóvenes de la Pontificia Universidad Católica del Ecuador", se concluyó que en la publicidad alrededor de 322 marcas utilizaban palabras del inglés, este uso le otorgaba a los productos distinción entre los demás, por eso el comportamiento de los consumidores hacia los productos era favorable. (Checa, 2018)

\section{PRÉSTAMOS LINGÜÍSTICOS}

Los anglicismos han sido adaptados directamente al español y sin su traducción , sucede esto, porque en el español no hay una palabra que guarde ese significado, software es una de ellas. (Ejemplos, 2019).

A través del tiempo las palabras pasaron a enriquecer el léxico de un pueblo, se desconoce en la historia un punto de inicio de este suceso, el registro de los anglicismos antiguos consta en el diccionario de Nebrija y los modernos en el Manual de Gramática Histórica Española. (Varela, 2009)

El Gran Diccionario de Anglicismos recopila el uso de expresiones del inglés que tratan sobre tecnología, deporte y música, aproximadamente 4500 entradas y que han sido revisadas recientemente.(Rodríguez, 2017)

Las investigaciones presentadas tienen los siguientes puntos de vista:

Halle en 1973 planteó un listado de morfemas que fueron utilizados para la formación de las palabras. El morfema especificaba la categoría gramatrical, esto restringía la selección de otra categoría. 
- $\quad$ M. Aronoff sobre la formación de las palabras destaco lo siguiente:

Un afijo se añade a las categorías gramaticales.

Una cualidad facultativa es la formación de las palabras.

La vocal última de una palabra se elimina y se añade un sufijo: marcha= marchista. (Bosque, Virtuniversidad.com, s.f.)

Según Poplack et al. (1984) un préstamo ('loanword) debe tener las siguientes características:

El uso frecuente y repetido de una palabra extranjera por un número considerable de hablantes permitirá el reconocimiento y utilización de la misma.

Un término foráneo desplaza a uno nativo y se lo considera como sinónimo.

Un préstamo toma los aspectos formales de una palabra como si fuese nativa.

La etimología de una palabra no es analizada e inconscientemente los hablantes aceptan un término.

Las diferentes etapas que un préstamo tiene que atravesar son:

El préstamo pasa a una lengua por el equivalente fonético más próximo.

El proceso de la acomodación es permitido por la lengua que es receptora. (Campbell, 1999)

- El uso de los nuevos préstamos es más repetitivo por el enlace cultural.(Castillo, 2002). En el Ecuador por ejemplo cuando se

2 Loanword $=$ préstamo, término utilizado por el autor Poplack et al. 1984: 99-100 tiene mucho calor se dice arrarray, esta palabra procede del kichwa.

- De la misma forma los galicismos han influenciado en el español, debido al contacto cultural.

- El uso de las palabras, especialmente en las redes sociales se ha convertido en un intercambio a discreción y entre todos los idiomas.

\section{PRÉSTAMOS LINGÜÍSTICOS DEL INGLÉS}

Los préstamos lingüísticos del inglés al español forman parte de varios campos del conocimiento, todos sabemos que la pasión de multitudes es el fútbol, esta disciplina deportiva utiliza un vocabulario considerable en los reportes periodísticos en la ciudad de Montevideo, Uruguay, en comparación con la computación, seguramente por su antigüedad. (La Paz, 2014). Este hecho similar se da en otros países de América Latina, los comentaristas deportivos ecuatorianos durante sus transmisiones utilizan el vocabulario futbolístico. En otros casos, el poder económico y político se ve reflejado en el intercambio no solo cultural, sino también comercial, los productos tienen nombres que son interiorizados por los consumidores en forma inconsciente y son utilizados como si pertenecieran a la lengua receptora, es el caso de la palabra axe que representa a un desodorante y que en el momento que se lo va a comprar nadie utiliza su traducción, es decir, hacha, siempre solicitan un axe. Esta influencia se presenta porque Estados Unidos es la primera potencia mundial que ha extendido su dominio económico, político y lingüístico en todas las naciones, el inglés es usado como idioma universal, por esta razón, quedarse al margen es atentar contra el progreso cultural, entonces, las decisiones políticas estatales se ven reflejadas en sus leyes, el artículo 80 del Reglamento de Régimen Académico del Consejo de Educación 
Superior (CES) del Ecuador, determinó como requisito de graduación, el aprendizaje de una segunda lengua. Los estudiantes de tercer nivel asisten con regularidad a clases de inglés, este hecho relacionado con la interactividad de las redes sociales ha permitido el uso de términos anglosajones en las diferentes actividades sociales. Por esta razón, las palabras del español y del inglés se han combinado, como por ejemplo música country y pie de manzana, etc.(Gerding, Cañete, \& Adam, 2018)

Una lengua de intercambio cultural podría ser superada en número si la RAE (Real Academia de la Lengua Española) relevara todos los anglicismos incorporados al español, en concordancia con los aportes de las academias de los países que la integran. La sincronía jugaría un papel importante en el registro de las palabras de una época determinada; por otro lado, la diacronía registraría todas aquellas palabras que se presentan a través del tiempo, estos diccionarios podrían cumplir un papel fundamental en el momento que se realicen, por ejemplo, traducciones, porque delimitarían las acepciones de las palabras de acuerdo a los diferentes contextos.

\section{EL PRÉSTAMO VERBAL}

Campbell 1999 manifestó que gracias a la Lingüística histórico-comparativa describe a través de la diacronía el préstamo verbal, esta se define como una palabra que se ha tomado de un idioma diferente y se lo ha incorporado con las adaptaciones pertinentes debido a su inexistencia. Este estudio se enmarca en el campo lingüístico de la morfología ${ }^{3}$ léxica porque versa sobre la formación de nuevas palabras. Así tenemos: tuitear de twit, loguear de log, chatear de chat, facebuquear ${ }^{4}$ de facebook etc. Estos verboides o formas no personales del verbo toman las diferentes desinencias del verbo, pertenecientes a la primera conjugación (ar), en los tiempos conocidos como presente, pasado y futuro. Las personas gramaticales de los verbos son seis: yo, tú, él, nosotros, vosotros y ellos ( con las respectivas formas femeninas para el = ella; nosotros =nosotras; vosotros = vosotras; ellos $=$ ellas), las primera tres personas corresponden al singular y las otras tres restantes al plural.

\begin{tabular}{l}
\hline 3 La morfología que pertenece a la gramática y esta \\
a la lingüística, se relaciona con hechos más complejas que \\
involucran a la sintaxis y a la semántica. \\
$4 \quad$ Este término es común en Ecuador, puede ser un \\
término nuevo en comparación con otros países.
\end{tabular}
término nuevo en comparación con otros países.

El siguiente ejemplo resume la relación paradigmática.

\begin{tabular}{|l|l|l|l|}
\hline & Presente & Pasado & Futuro \\
\hline Yo & logue-o & logue-é & \\
\hline Tú & logue-as & logue-aste & logue-aré \\
\hline Él, ella & logue-a & logue-ó & logue-arás \\
\hline Nosotros /as & clogue-amos & logue-amos & logue-aremos \\
\hline Vosotros /as & logue-áis & logue-asteis & logue-aréis \\
\hline Ellos/as & logue-an & logue-aron & logue-arán \\
\hline
\end{tabular}


En base a lo mencionado, el préstamo verbal lo enmarcan dentro de las normas de la primera conjugación y además la misma categoría gramatical con las desinencias presentadas; este proceso para el hablante se vuelve automático e inconsciente, es decir, vuelve a aplicar de la misma forma el procedimiento con otros préstamos.

\section{REFERENCIAS BIBLIOGRAFICAS}

Bosque, I. (s.f.). Virtuniversidad. com. Obtenido de https://www. virtuniversidad.com/greenstone/ collect/ingles/import/CuatrimestreV/ Morfolog \% C 3\% AD a \% 20 y \% 20 Sintaxis $\% 201 /$ morfolog\%C3\%ADa\%20 sincr\%C3\%B3nica.pdf

Brochier, G. (6 de Octubre de 2019). Normativa de los préstamos lingüísticos. $(\mathrm{H}$. Romero, Entrevistador)

Checa, G. (25 de 10 de 2018). Repositorio de Tesis de Grado y Posgrado. Obtenido de http://repositorio.puce.edu.ec/ handle/22000/15355

Cuéllar, L., \& Izurieta, P. (2017). Repositorio Digital. Obtenido de http://www.dspace. uce.edu.ec/handle/25000/12836

Gerding, C., Cañete, P., \& Adam, C. (Agosto de 2018). Revista Signos. Obtenido de http://www.revistasignos.cl/index.php/ signos/article/view/202/90

Gerding, C., Fuentes, M., Gómez, L., \& Kotz, G. (Junio de 2014). Scielo. Obtenido de http://www.scielo.org.co/scielo. php?script=sci_arttext\&pid=S0123$46412014000100005 \&$ lang=es

Rodríguez, F. (2017). Gran diccionario de anglicismos. Madrid: Arco Libros.
Yoza, N. (Mayo de 2013). Academia. Obtenido de https://www.academia. edu/5913400/Pr\%C3\%A9stamos_ de_\%C3\%ADtemes_I\%C3\%A9xicos_ del_ingl\%C3\%A9s_al_castellano_ formaci\%C3\%B3n_de_neologismos_ en_-ear_Tesis_de_licenciatura_ 